\title{
Desiguales ante la muerte. Un estudio de caso en el Sur de España (1850-1950) ${ }^{1}$
}

\section{Unequal in the face of death. A case study in Southern Spain (1850-1950)}

\author{
Víctor A. LUQue dE HARO \\ Universidad de Almería \\ vld417@ual.es \\ https://orcid.org/0000-0001-9521-253X \\ Andrés SÁnchez PIcón \\ Universidad de Almería \\ aspicon@ual.es \\ https://orcid.org/0000-0002-9401-1741
}

Fecha de recepción: 20-06-2019

Fecha de aceptación: 17-11-2019

\section{RESUMEN}

El presente trabajo analiza las diferencias sociales en la mortalidad, poniendo en relación el desarrollo económico y las condiciones de vida de la población a partir del estudio de un caso: Vera, una localidad del sudeste español. La mayor parte de los datos se han extraído de censos de población y libros de defunciones parroquiales. La relación entre las tasas de mortalidad estandarizadas de cada uno de los grupos socioeconómicos ha sido el principal indicador utilizado para observar las diferencias en las condiciones de vida entre categorías ocupacionales. Los resultados muestran una progresiva reducción de la desigualdad social ante la muerte entre la población adulta desde el año 1860, momento en el que comienza nuestro análisis, hasta los años 30 del siglo XX. Este proceso de convergencia se trunca dramáticamente en los años de la posguerra. El comportamiento de la mortalidad desagregado por categorías socioeconómicas parece estar en línea con la evolución de los factores nutricionales y los relacionados con los avances higiénicos y en la salud pública.

Palabras clave: desigualdad, benestar demográfico, demografía histórica, historia económica, transición demográfica.

Topónimos: Andalucía

Periodo: siglos XIX y XX

1 Este trabajo ha sido financiado por el Ministerio de Ciencia, Innovación y Universidades, a través del proyecto PGC2018-097817-B-C32 


\section{ABSTRACT}

This work analyses social inequality in relation to the demographic living conditions of the population, examining the relationship between economic development and mortality based on a case study: Vera, a town in southeast Spain. Most of the data was taken from censuses, population registers and parish burial records. The relationship between the standardized mortality rates of each socioeconomic group was the main indicator used to observe the differences in living conditions between occupational categories. The results show a progressive reduction of social inequality in mortality among adults between 1860 and the 1930s. This process of convergence is truncated dramatically in the postwar years. The behaviour of mortality disaggregated by socioeconomic categories seems to be in line with the evolution of nutritional factors and those related to hygienic advances and public health.

Key words: inequality, demographic welfare, demographic history, economic history, demographic transition.

Toponyms: Andalusia

Period: $19^{\text {th }}$ and $20^{\text {th }}$ centuries

\section{INTRODUCCIÓN}

El estudio de la evolución del nivel de vida ha sido una sido una constante en el ámbito de la historia económica desde su nacimiento como disciplina y un elemento de notable interés para la historia social. Los trabajos sobre el nivel de vida en España a partir de los niveles de mortalidad durante los siglos XIX y XX suelen incluir argumentaciones sobre diferencias sociales. Esto se evidencia en los relacionados con la urban penalty ${ }^{2}$, en los que se centran en el análisis de las diferencias regionales en la mortalidad ${ }^{3}$ y en aquellos cuyo objeto son las diferencias por barrios dentro de las ciudades ${ }^{4}$. Sin embargo, la manera en la que la mayoría de los estudios en España han tratado o recogido los datos ${ }^{5}$, ha conllevado unos resultados que, sin perjuicio de su utilidad para el análisis de las tendencias del conjunto de la población, no permiten el cálculo de las diferencias entre categorías socioeconómicas dentro de esas unidades territoriales (Bengtsson, 2004).

Este trabajo tiene como objetivo el análisis del impacto diferencial sobre los niveles de mortalidad que tuvieron el desarrollo económico y las distintas transformaciones producidas entre 1850 y 1950, en la ciudad andaluza de Vera, una localidad del sudeste español. La principal novedad respecto a otras aportaciones reside en la introducción de la diferenciación por grupos socioeconómicos.

La elección de los años objeto de estudio obedece a que durante ese periodo se produjeron en España las principales transformaciones demográficas, del patrón epidemiológico y en materia de atención sanitaria (Pérez Moreda, Reher y Sanz Gimeno, 2015; Robles González, García Benavides y Bernabeu-Mestre, 1996). Esta perspectiva de

2 Para una mayor profundización en el fenómeno de la penalización urbana en España ver Escudero y Nicolau (2014); García Gómez (2016); Reher (2001); Revuelta Eugercios y Ramiro-Fariñas (2016).

3 Ver Domínguez y Guijarro (2000) y Sánchez Aguilera (1996). Sí que encontramos algunos estudios que analizan las desigualdades socioeconómicas en la actualidad a partir de los niveles educativos. Sin embargo, son relativamente escasos y centran su análisis en las últimas décadas del siglo XX o la primera del XXI (Reques et al., 2015).

4 Ver Kesztenbaum y Rosenthal (2016) y Recaño y Esteve (2006).

5 En numerosas ocasiones los vaciados no han sido completos sino parciales. 
largo plazo permite analizar la evolución de la mortalidad para cada colectivo y el impacto diferencial que los cambios institucionales, económicos, políticos y sociales tuvieron sobre ellos. Además, posibilita la distinción entre los cambios de tendencia y aquellos que son coyunturales, ya que, como consecuencia de la transición demográfica y epidemiológica, la mortalidad y la recurrencia de epidemias disminuyó notablemente durante el periodo analizado (Livi Bacci, 1990).

La elección de la ciudad o municipio como dimensión territorial nos permite analizar, de forma más adecuada que las regiones administrativas o históricas, las diferencias y similitudes que se produjeron en la transición de la mortalidad en España y su impacto sobre la evolución de los indicadores del bienestar en los diferentes modelos productivos (Parejo, 2006). En el ámbito geográfico local se identifica con mayor precisión el comportamiento de los determinantes de la mortalidad sobre los diferentes grupos socioeconómicos y, por tanto, el impacto diferencial de estos sobre las condiciones de vida (Bengtsson et al., 2004). Además, la necesidad de contabilizar individualmente el número de vecinos pertenecientes a cada categoría ocupacional para analizar las desigualdades sociales ha sido otro de los factores que nos ha influido en la elección de esta dimensión territorial.

Nuestro conocimiento sobre el comportamiento de las desigualdades sociales en mortalidad a largo plazo se basa, principalmente, en la historiografía británica, debido al papel protagonista que ha ocupado Inglaterra en el estudio de los niveles de bienestar y las condiciones de vida de la población a partir de la Revolución Industrial. No obstante, se debe advertir que las singularidades del desarrollo económico experimentado por dicho territorio no son extrapolables al resto de países (Bengtsson y Van Poppel, 2011). Por esta razón, el avance en el estudio sobre la evolución del nivel de vida a nivel histórico en los distintos países pasa por la realización de estudios de caso en los mismos.

En relación con la evolución en el largo plazo de las diferencias socioeconómicas en la mortalidad, en el ámbito académico se plantean dos hipótesis principales: la teoría de la constancia y la hipótesis de la divergencia-convergencia. Tradicionalmente se ha tendido a pensar que las desigualdades en la mortalidad por clases sociales habían existido siempre. Así, Marmot (2004: 150) afirma que "la salud y la longevidad están íntimamente relacionadas con la posición en la jerarquía social" y que "cuanto menor es el estatus, mayor es el riesgo de enfermedad y muerte y, consecuentemente, menor la esperanza de vida". Link y Phelan $(1995,1996,2002)$ afirman que esa desigualdad ha existido en todo tiempo y lugar y defienden que las diferencias por clases sociales incluyen recursos como educación, dinero, poder y prestigio, entre otros factores, que a su vez permiten reducir riesgos de contraer enfermedades y minimizar sus consecuencias una vez contraídas. Estos recursos pueden ser usados de forma diferente en distintas situaciones y son capaces de proporcionar una ventaja incluso cuando el perfil de los factores de riesgo ha ido cambiando continuamente. Esta interpretación, conocida como Teoría de la causa fundamental, ha sido combinada con elementos de la teoría de la transición demográfica y de la epidemiológica para explicar el desarrollo de la desigualdad en causas de mortalidad específicas (Clouston et al., 2016). De acuerdo con estos autores, los niveles de desigualdad pasan por distintas fases conforme avanza el conocimiento médico sobre la enfermedad en cuestión.

La otra hipótesis se denomina "divergencia-convergencia" (Bengtsson y Van Poppel, 2011). Antonovsky (1967) sostiene que en un principio las diferencias de mortalidad eran reducidas puesto que la facilidad de contagio de las enfermedades y su alta virulencia, debida a la ausencia de tratamientos adecuados, hacía que la mortalidad no estuviera ligada a un componente social como el nivel de nutrición. En una segunda fase (desde el siglo XVIII 
a la segunda mitad del XIX) aparece una brecha en los niveles de mortalidad entre clases sociales como consecuencia de un comportamiento dual: una mejora notable del nivel de vida entre las clases más acomodadas y un estancamiento o incluso empeoramiento en las condiciones de vida de las clases bajas con el surgimiento de la figura del proletariado en las grandes ciudades. Finalmente, en una tercera fase, que se desarrolla a partir de la segunda mitad del siglo XIX, las diferencias vuelven a reducirse. Sin embargo, Antonovsky (1967) alerta de la posibilidad de que estas desigualdades vuelvan a ampliarse como consecuencia de las mejoras médicas y de la consolidación de las enfermedades crónicas como principal causa de defunción en las sociedades más desarrolladas.

En los últimos años nos encontramos con diversos estudios internacionales que abordan la cuestión de las diferencias en la mortalidad por categorías socioprofesionales en distintos países y momentos, con resultados poco coincidentes. Algunos confirman la hipótesis de la convergencia (Schumacher y Oris, 2011) y otros muestran una suerte de alternancia entre periodos de convergencia y divergencia (Breschi, Fornasin, Manfredini, Mazzoni, y Pozzi, 2011; Haines, 2001). Un tercer grupo encuentra que las diferencias en mortalidad no fueron observables hasta finales del siglo XIX o principios del siglo XX (Schenk y Van Poppel, 2011; Edvinsson y Broström, 2017). El incremento de las diferencias sociales en mortalidad durante el siglo XX ha llevado a algunos autores a renombrar la hipótesis como hipótesis de la divergencia-convergencia-divergencia (Bengtsson y Van Poppel, 2011). Los resultados de estos trabajos muestran que las diferencias no han sido similares siempre y en todo lugar, sino que dependen de las singularidades de las distintas economías, sus procesos de desarrollo, el estado de la ciencia médica y del patrón epidemiológico predominante.

\section{EI ÁREA DE ESTUDIO: LA CIUDAD DE VERA}

La elección de la ciudad de Vera (Almería) como objeto de estudio, se debe a distintas razones entre las que destaca la calidad de sus fuentes archivísticas ${ }^{6}$. Además, el tamaño de su población, en el intervalo entre 1.000 y 10.000 habitantes durante todo el periodo de estudio, la hace representativa de la demografía española de finales del siglo XIX, en la medida en que la mayoría de la población española vivía en municipios de este tamaño (Zoido y Arroyo, 2003). La economía de Vera estaba compuesta por sectores como la agricultura, la pesca, la artesanía o la minería, que presentaron una importancia heterogénea y cambiante durante el siglo que analizamos. En cualquier caso, debe destacarse la influencia que tuvo en el desarrollo de la ciudad y de la minería española del siglo XIX el florecimiento de la actividad minera en la vecina Sierra de Almagrera tras el fulgurante hallazgo de un filón de plomo argentífero en 1839 (Sánchez Picón, 2011).

A pesar de la repercusión del desarrollo minero desde 1840, el modelo minero de la zona resultaba ambiguo en la configuración del mercado de trabajo. La proletarización parcial de los mineros (al compaginar el trabajo minero con las labores agrícolas) se manifiesta en el mantenimiento de una estructura de la población activa bastante tradicional. Las actividades productivas del sector agrícola siguieron siendo predominantes en términos de empleo (Sánchez Picón, 1992) y de hecho, la entrada de capitales mineros tuvo un efecto virtuoso en la transformación de la agricultura de la zona favoreciendo la implantación de nuevos cultivos orientados a la exportación. El papel protagonista de la agricultura en la economía de la ciudad queda reflejado en el informe que el Banco de España (1900: 27-29) elaboró

6 Al contrario que en la mayoría de los municipios, en la localidad de Vera, se encuentran los libros censales completos, un registro sistemático de los fallecidos durante el periodo estudiado y, además, la mayoría de los libros de defunciones y los censos incluyen tanto la edad como la ocupación de los individuos. 
para analizar la ubicación idónea de una nueva sucursal en la región norte de la provincia: "La producción agrícola es la más importante, pues, además de producir cereales bastantes para su consumo, tiene otros frutos de los que hace objeto de grandes exportaciones, como son: la naranja, los higos, las almendras y algarrobas y la uva de embarque".

Durante el periodo que analizamos (1850-1950) la población media del municipio es de unos 7015 habitantes. El máximo poblacional documentado se alcanza en 1877 con 8.796 residentes mientras que el mínimo se documenta en el censo de 1950, con 4.700 habitantes.

\section{FUENTES, DATOS Y METODOLOGÍA}

A continuación, se describirán los aspectos metodológicos más importantes relacionados con las fuentes de las que se han extraído los datos, la forma en que se han realizado las agrupaciones socio-profesionales y el procedimiento utilizado para el cálculo de las diferencias en mortalidad entre las mismas.

Los documentos de donde se ha extraído el grueso de la información han sido los censos de población, para la distribución de la estructura social (en concreto, el padrón de 1860 y los censos de 1900, 1910, 1930, 1940 y 1950), los libros de defunciones parroquiales para la información sobre los fallecidos (1858-1861; 1899-1903; 1908-1912; 1927-1933; 1939-1942 y 1947-1953). En total se han tabulado los datos de más de 53.000 individuos (6.716 registros de fallecidos y 46.678 registros de individuos en censos) en los cuales se han incluido las siguientes variables:

- En relación con la estructura de la población (censos): edad, sexo, ocupación y alfabetización.

- Respecto a los fallecidos (libros de defunciones parroquiales): edad, sexo, ocupación y causa de muerte.

Para la agrupación por categorías profesionales hemos usado la Historical International Standard Classification of Occupations (HISCO) 7 . A partir de los distintos códigos que HISCO asigna a cada ocupación, hemos utilizado el esquema HISCLASS ${ }^{8}$ para estructurarlos en grupos sociales. Esta elección nos permite que los resultados sean más fácilmente comparables. Para realizar los análisis comparativos y los cálculos de desigualad, hemos agrupado las categorías 1-7 como clase social media-alta y las categorías 8 a la 12 como clase social baja.

A continuación, mostramos la estructura ocupacional de la población censal en los distintos subperiodos analizados. Como podemos observar en todos los años, más de un $40 \%$ de la población figura en los registros sin ocupación, por lo que no ha sido posible incluirlos en ninguna categoría HISCLASS. En el caso de las mujeres, debido a la omisión generalizada de sus ocupaciones en los censos (Sarasua, 2019), se cuenta con un porcentaje de información muy reducido, con la excepción de 1860. En la mayoría de ellas figura "su sexo" o "sus labores" como ocupación. Este cambio no se debe tanto a un cambio de realidad, en el que la mujer abandona la actividad laboral, sino que más bien es reflejo de una ideología "bajo la cual la mujer casada es madre y esposa, pero no ejerce un trabajo remunerado fuera del hogar" (Pareja Alonso, 2006: 203). Esta circunstancia provoca que los

7 Ver Pujadas Mora, Romero Marín, y Villar Garruta (2014) y Van Leeuwen, Maas y Miles (2002).

8 HISCLASS es una clasificación histórica creada con el propósito de hacer comparaciones entre diferentes países y en diferentes periodos. Clasifica las ocupaciones en clases sociales intentando reflejar condiciones de vida y posibilidades similares en los distintos grupos y diferentes entre ellos. Ver Van Leeuwen y Maas, (2011). 
resultados sobre desigualdad entre categorías socioeconómicas se refieran exclusivamente a los varones no debiendo extrapolarse las conclusiones al sexo femenino.

Tabla 1. Estructura ocupacional en Vera (individuos de 15 años o más)

\begin{tabular}{|c|c|c|c|c|c|c|c|}
\hline & 1860 & 1900 & 1910 & 1930 & 1940 & 1950 & Total \\
\hline $\begin{array}{c}\text { Categoría HISCLASS } \\
\text { media-alta }\end{array}$ & $14^{\prime} 9$ & $17^{\prime} 9$ & $14{ }^{\prime} 7$ & $16{ }^{\prime} 2$ & $18^{\prime} 2$ & $19 ' 9$ & 4308 \\
\hline 1 & $3{ }^{\prime} 3$ & $6 ' 2$ & $2 ’ 1$ & $3 ’ 1$ & 3 '8 & 2'8 & 934 \\
\hline 2 & 1'8 & $2{ }^{\prime} 6$ & $2 ' 4$ & $3 ' 2$ & $5 ’ 6$ & $4 ' 9$ & 843 \\
\hline 3 & $0 \prime 1$ & 0 & $0 ’ 1$ & $0 ’ 1$ & $0 ’ 1$ & $0 ’ 1$ & 21 \\
\hline 4 & $1 ' 7$ & $2{ }^{\prime} 0$ & $3 \prime 1$ & $2 ' 9$ & $1 ' 9$ & 4 '1 & 669 \\
\hline 5 & $1 ' 9$ & $2{ }^{\prime} 0$ & $1 ' 6$ & $2 ' 4$ & $3{ }^{\prime} 3$ & 27 & 576 \\
\hline 6 & 0 '0 & $0 ’ 1$ & $0 ’ 5$ & $00^{\prime}$ & $00^{\prime}$ & $00^{\prime}$ & 35 \\
\hline 7 & $6{ }^{\prime} 0$ & $4^{\prime} 9$ & $4{ }^{\prime} 8$ & $4 ' 4$ & $3 ' 5$ & $5 \cdot 2$ & 1230 \\
\hline Categoría HISCLASS baja & $42 ' 1$ & $32^{\prime} 9$ & $32 ' 8$ & $31 ’ 8$ & $2^{\prime}, 7$ & $27 ’ 1$ & 8399 \\
\hline 8 & $1^{\prime} 2$ & $0 ’ 3$ & 0 & 0'1 & 0 '2 & $0 ' 4$ & 91 \\
\hline 9 & 9'6 & $1 ' 4$ & 0 '9 & $2 ' 5$ & 3'1 & $2 ' 8$ & 786 \\
\hline 10 & 3 '3 & 2'1 & $2 ' 0$ & $3 ' 4$ & $3 ' 6$ & $4^{\prime} 7$ & 770 \\
\hline 11 & 8'3 & $3 ’ 5$ & 0 '4 & $1 ' 3$ & $1 ' 9$ & $2 ' 5$ & 727 \\
\hline 12 & $19^{\prime} 7$ & $25 ’ 5$ & $29 ' 5$ & $24{ }^{\prime} 4$ & $20 ’ 9$ & $16^{\prime} 7$ & 6025 \\
\hline Sin ocupación HISCLASS & $43^{\prime} 0$ & $49^{\prime} 2$ & $52 ' 5$ & $52 ' 1$ & $52 ' 2$ & $53{ }^{\prime} 0$ & 12904 \\
\hline "Sus labores" & $2 ' 2$ & $48^{\prime} 9$ & $51 ’ 2$ & $51^{\prime} 6$ & $51^{\prime} 6$ & 507 & 11209 \\
\hline \#N/A & $40 ’ 8$ & 0,3 & $1^{\prime} 2$ & $0 ’ 4$ & 0'6 & $2 ' 3$ & 1695 \\
\hline Total & 3660 & 5520 & 5569 & 3799 & 3620 & 3443 & 25611 \\
\hline
\end{tabular}

Fuente: Elaboración propia a partir de los libros censales. Datos en \% excepto en la fila y columna "Total" en la que se muestran valores absolutos.

El protagonismo del sector agrario en la economía de la ciudad se comprueba al analizar las ocupaciones que predominan en cada categoría. En las clasificadas como clase media-alta encontramos principalmente propietarios de tierras en la categoría 1 (56 \% de la misma), profesionales en la categoría 2, comerciantes y barberos en la 4, empleados en la 5 (más de un $50 \%$ y albañil, zapatero, alpargatero y carpintero en la 7 (más de un $55 \%$ 
del total de la categoría). En las categorías clasificadas como clase baja, sobresale la figura del jornalero (5.830) dentro de la categoría 12, que incluye a los trabajadores del campo no cualificados. De una importancia relativa menor, aunque también considerable, destacan las ocupaciones de arriero (99), labrador (693) y sirvientas y criadas (364) pertenecientes a las categorías 9, 10 y 11 respectivamente ${ }^{9}$.

En este trabajo hemos optado por el uso de distintos indicadores que nos permitieran abordar una perspectiva de largo plazo, sin acudir al laborioso proceso de la reconstrucción familiar mediante la utilización de la vinculación nominativa de individuos o record linkage ${ }^{10}$ (Reher, 2000: 32). A continuación, describiremos brevemente las opciones metodológicas que se nos presentaban, las ventajas y desventajas de cada una de ellas, así como la bibliografía consultada.

En primer lugar, se planteó la posibilidad de acercarnos a la posible existencia de sobremortalidad entre estratos sociales a partir de la edad media de fallecimiento, debido a la facilidad de su cálculo. Este indicador ha sido usado en diferentes estudios (Clark, 2008; Kesztenbaum y Rosenthal, 2016). Sin embargo, presenta una evidente limitación, al no contemplar la forma en que se distribuye la población por edades en cada una de las categorías socioeconómicas. Otro indicador que descartamos fue el de la esperanza de vida al nacimiento para cada una de las categorías socioeconómicas. Lo hemos realizado para la población en su conjunto (gráfico 2), a partir del método de la tabla de vida actuarial abreviada (Vázquez et al., 2003). No obstante, el tamaño de la población no nos ha permitido descender entre categorías socioeconómicas y ofrecer unos resultados suficientemente robustos.

También se barajó la opción de utilizar el índice de Gini para el cálculo de la desigualdad en la salud, en sintonía con numerosos trabajos (Llorca, Prieto Salceda y Delgado-Rodríguez, 2000; Pérez de Perceval Verde, Martínez-Carrión y Martínez-Soto, 2016). Este indicador presenta varias ventajas: en primer lugar, incluye a todos los individuos en el cálculo y no solo aquellos para los que contamos con ocupación; y, en segundo lugar, elimina los problemas asociados con la distribución ocupacional, en la medida en que no diferencia entre clases sociales (Llorca et al., 2000). Sin embargo, esta última característica lleva aparejada un inconveniente puesto que no permite capturar las desigualdades en la mortalidad entre las mismas (Wagstaff, Paci, y van Doorslaer, 1991). En cualquier caso, finalmente hemos creído conveniente calcular los valores de este indicador en los distintos subperiodos, puesto que refleja con nitidez uno de los rasgos más característicos de la superación del

9 Esta clasificación no está exenta de controversias, especialmente en relación con la adscripción de determinadas ocupaciones a distintas categorías socioeconómicas. En este sentido, las características asociadas a la figura del "labrador" han sido objeto de cuantioso debate por la historiografía (Artola, Bernal, y Contreras, 1978; Cámara Hueso, 2007; Martínez-Carrión y Pérez Castejón, 2002). Sin embargo, como ha señalado Mignon (1982), en la montaña andaluza la pequeña explotación campesina tradicional ha aprovechado históricamente un suelo tan mediocre que las familias han debido de utilizar tradicionalmente recursos complementarios como la emigración estacional (Sánchez Picón, 1988). Además, en el caso concreto de Vera, los datos del Amillaramiento de 1860 ofrecen una imagen clara de la vulnerabilidad económica de este grupo social (Archivo Municipal de Vera, leg. 206-6-3) y los registros censales muestran que su tasa de alfabetización se asimila a la presentada por los jornaleros y a mucha distancia de la de los propietarios y los empleados. Por estos motivos, hemos decidido mantenerlos entre las categorías que hemos considerado como "clase baja", respetando la codificación de HISCO propuesta para el caso español por el grupo de investigación Trabajo, Instituciones y Género de la Universidad de Barcelona. Así, hemos clasificado a los que figuraban como labradores o labradores medieros con el código 61110 - Explotadores agrícolas en general (HISCLASS 8) y a los que figuraban como labradores de tierras ajenas o labradores arrendatarios con el código 61115 - Labrador (subsistencia) (HISCLASS 10).

10 Para profundizar en record linkage ver Winkler (1999) 
régimen demográfico antiguo: la caída en la mortalidad infantil y el consiguiente aumento de la supervivencia de un elevado porcentaje de la población hasta la vejez.

La tasa bruta de mortalidad para distintas categorías profesionales ha sido otro indicador utilizado en el estudio de las diferencias de mortalidad (Williamson, 2002). Sin embargo, las comparaciones de mortalidades a partir del mismo pueden ser engañosas ya que las diferencias pueden imputarse no solo a la intensidad del fenómeno sino también a la estructura de edades de la población (Livi Bacci, 1993: 99-103; Pressat, 1993: 143). Esta característica puede llevar al error de considerar que las condiciones de vida asociadas a una categoría profesional son peores que las de otra, cuando dicho resultado se podría deber a la mayor edad media de dicha categoría. Este fenómeno se conoce en demografía como efecto edad y se refiere a la variación en la probabilidad de que se produzca un evento como consecuencia de la edad (Elder y Pellerin, 1998).

Para salvar este inconveniente hemos optado por calcular las tasas de mortalidad específicas y estandarizarlas posteriormente para posibilitar la comparación entre sí (Pamuk, 1985; Reques et al., 2015)ํㅜ. Así, hemos utilizado la estructura de población típica de España en 1900 para proceder a la estandarización, utilizando solo la población mayor de 15 años, puesto que es para la que disponemos de información sobre ocupaciones. La elección de la población de España nos permite contrastar los resultados con los contenidos en otros estudios. Adviértase, además, que el año 1900 ocupa un lugar intermedio en nuestro periodo de estudio.

Por lo que respecta a los subperiodos, se ha optado por analizar aquellos para los que se contaba con censo que incluyera información sobre las edades y ocupaciones de la población. Los cálculos de las tasas específicas por edad para cada una de las categorías ocupacionales se han realizado utilizando como población la del año censal y como número de difuntos el correspondiente a las defunciones medias del subperiodo a la edad $x$ de las distintas categorías. El cálculo de la tasa específica de la edad x y la categoría HISCLASS i para un subperiodo en el que utilicemos 5 años de fallecidos se haría de la siguiente manera:

$$
t_{x i}=\frac{\left.\left(\mathrm{M}_{\mathrm{xit}-2}+\mathrm{M}_{\mathrm{xit}-1}+\mathrm{M}_{\mathrm{xit}}+\mathrm{M}_{\mathrm{xit}+1}+\mathrm{M}_{\mathrm{xit}+2}\right) / \mathrm{n}\right)}{\mathrm{P}_{\mathrm{xit}}}
$$

Donde $\mathrm{M}_{\text {xit }}$ es el número de fallecidos de la edad $x$ y categoría socioeconómica baja en el periodo $t$. $\mathrm{P}_{\text {xit }}$ representa la población de edad $x$ y categoría $i$ del año considerado y $n$ el número de años del subperiodo.

De la misma manera se calcula la tasa específica de la edad x para la categoría media-alta, utilizando los datos de fallecimientos y población. A continuación, se procede a su comparación mediante la ratio de la tasa de mortalidad estandarizada de las categorías ocupacionales que hemos agrupado como "clase baja" entre la de las agrupadas como "clase media y alta". El resultado será un índice de la sobremortalidad existente en el subperiodo:

$$
\text { Índice de sobremortalidad }=\frac{\Sigma p^{\prime \prime} x^{*} t_{x i}}{\Sigma p^{\prime \prime} x^{*} t_{x i}}
$$

11 Sobre la estandarización con el método de la población tipo ver Livi Bacci (1993: 99-103) 
Donde $p^{\prime \prime}$ es la proporción de individuos de la edad $x$ en la población española de 1900; $t_{x i}$ es la tasa de mortalidad específica de edad x para la categoría baja, y t es la tasa de mortalidad específica de edad x para la categoría media-alta.

Al hacer análisis transversales de la mortalidad, no observamos de forma completa el comportamiento de las diferentes cohortes o generaciones a lo largo del tiempo (análisis longitudinal). Sin embargo, el carácter particular del fenómeno mortalidad y la menor dependencia de las manifestaciones de mortalidad de un año respecto a los anteriores, al contrario de lo que sucede con otras variables demográficas como la nupcialidad o la fecundidad, justifican la utilización de este tipo de análisis (Pressat, 1993: 132).

La comparación entre tasas estandarizadas consideramos que presenta evidentes ventajas en relación con aquellos trabajos que utilizan indicadores más simples, como puede ser la edad media de fallecimiento, ya que los cálculos incorporan la distribución de la población por edades y por clases sociales. Por otro lado, comparando nuestra metodología con la de los análisis de supervivencia a partir de microdatos individuales en forma de datos longitudinales, la principal ventaja es que conlleva un importante ahorro de trabajo y de tiempo, en la medida que no exige realizar ni un vaciado nominativo del conjunto de los individuos, ni una vinculación entre los mismos, ni una limpieza de los datos (Bengtsson, 2004: 14; Reher, 2000: 32-36).

Igualmente, estudiar las diferencias mortalidad a partir del número de individuos en cada categoría profesional, tanto en censos como en registros de defunciones, puede presentar problemas. Piénsese que todas las personas que computamos en el denominador no tienen que estar necesariamente en el numerador y viceversa, ya que las defunciones se registran en el lugar donde ocurrieron en vez de en el lugar de residencia (Revuelta Eugercios y Ramiro-Fariñas, 2016). Por otra parte, el uso de esta metodología no nos permite observar la influencia de la movilidad social en los niveles de mortalidad (Fox et al., 1985). En todo caso, nuestros resultados, a partir de las mediciones puntuales de los niveles de mortalidad, permitirán observar la tendencia experimentada por cada una de las categorías socioeconómicas. Sin embargo, un estudio a partir de microdatos longitudinales que incluya variables suficientes y significativas, proporcionaría resultados más precisos y posibilitaría un análisis específico sobre las relaciones causales entre dichas variables y la mortalidad.

\section{EVOLUCIÓN DE LA MORTALIDAD Y LA ESPERANZA DE VIDA}

Previamente a la presentación de la desagregación de las tasas de mortalidad por categorías profesionales, comparamos los datos agregados de mortalidad y de esperanza de vida de Vera con los de las distintas divisiones administrativas de las que forma parte (Almería, Andalucía, España).

En el siguiente gráfico podemos observar la evolución de las tasas de mortalidad. La tendencia del indicador es similar para los distintos territorios. Se produce un estancamiento, o incluso un empeoramiento, en los valores desde el comienzo de la serie (1850) hasta aproximadamente la década de 1890. Desde ese momento, se aprecia una mejora continuada, que solo se ve truncada por diferentes crisis de mortalidad. La mayor variabilidad de los datos de Vera, especialmente respecto a Andalucía y a España, se debe al menor tamaño de la ciudad. 
Gráfico 1. Tasa bruta de mortalidad (TBM) en Vera, Almería, Andalucía y España

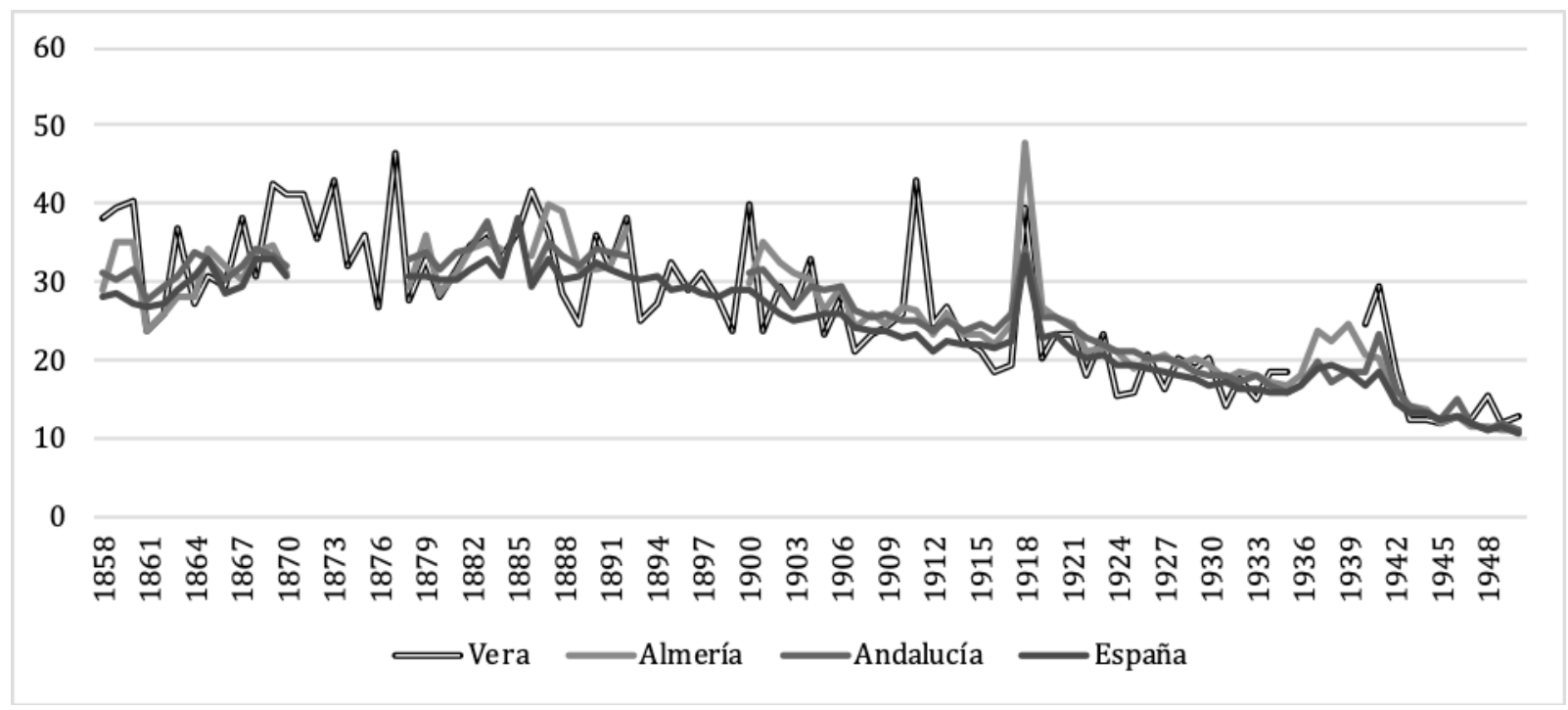

Fuentes: Vera: elaboración propia a partir de los censos, padrones y los libros de defunciones parroquiales; Almería y Andalucía: Instituto de Estadística y Cartografía de Andalucía (2004); España: para los años 1858 a 1870 Nadal (1984). Desde 1878 en adelante Nicolau (2005). TBM no estandarizadas en \%.

Desde el comienzo de la serie hasta el pico de fallecimientos provocado por la Gripe de 1918 existen variaciones abruptas en los niveles de mortalidad reflejo de la recurrente incidencia de epidemias (González García, 2013). Sin embargo, entre esa fecha y el final de la serie, vemos que no se produce ningún episodio de mortalidad catastrófica de magnitud similar a los anteriores. Los únicos años en los que volvemos a observar un fuerte incremento en la mortalidad (1939-1942) coinciden con la posguerra española. Durante la primera mitad del siglo XX los datos de mortalidad de Vera experimentaron las transformaciones propias del paso del régimen demográfico antiguo al régimen demográfico moderno. Ello conllevó una reducción sustancial de los niveles de mortalidad y la práctica desaparición de los episodios recurrentes de mortalidad epidémica.

En el gráfico 2 está representada la comparación de los niveles de esperanza de vida en Vera con los de la provincia de Almería, la región Andaluza y los valores agregados de España. La tendencia que se observa en la esperanza de vida al nacer en Vera es similar a la experimentada en Almería, Andalucía y España. Se comprueba una mejora en los valores de este indicador en todos los subperiodos, con la excepción de la caída producida en los años inmediatamente posteriores a la Guerra Civil Española. 
Gráfico 2. Esperanza de vida al nacimiento en Vera, Almería, Andalucía y España

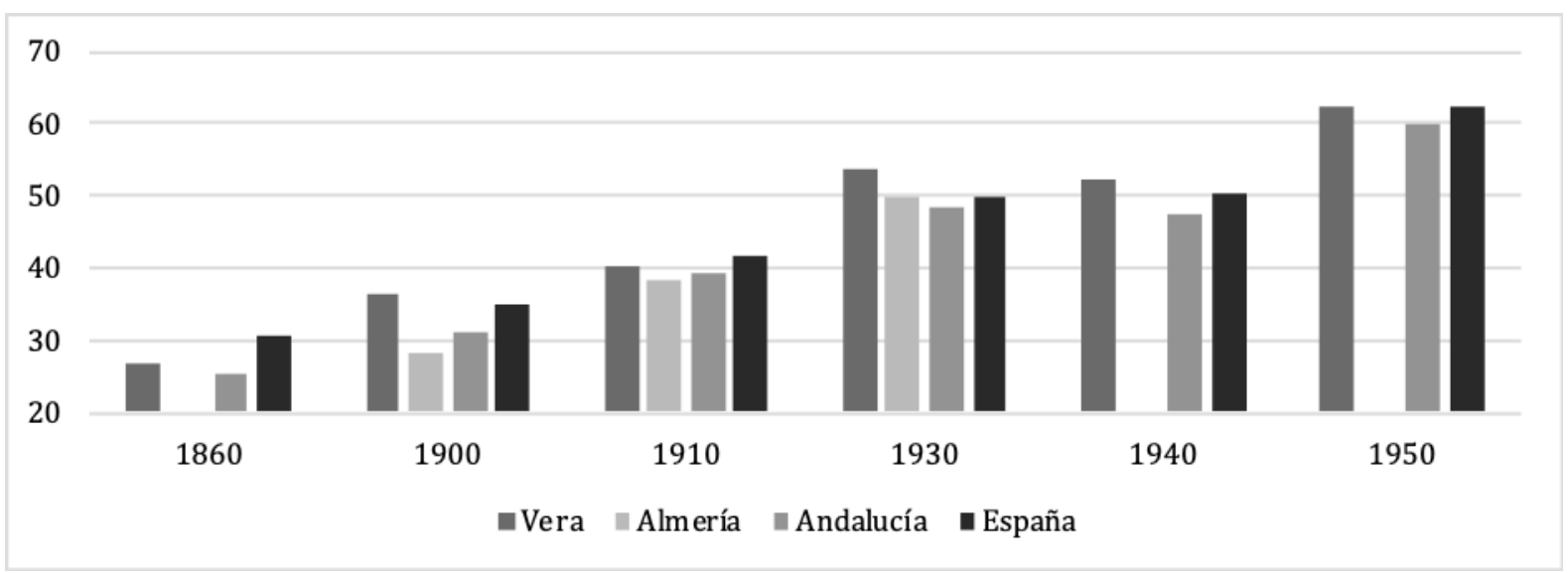

Fuente: Vera: elaboración propia a partir de los libros censales y los registros parroquiales; España y Andalucía 1860: Muñoz Pradas (2005); España, Almería y Andalucía 1900-1930: Dopico y Reher (1998);

Andalucía 1940-1950: Calot (1999); España 1940-1950: Goerlich Gisbert y Pinilla Pallejà (2006).

Los valores de Vera son bastante similares a los del conjunto de España. Sin embargo, son algo superiores a los de Almería y Andalucía, en la mayoría de los periodos, especialmente desde 1900 a 1930. Esto es coherente con los diferenciales en la mortalidad entre las capitales de provincias y el resto de municipios de las mismas, observados por Dopico y Reher (1998). Ya que las referencias de Almería, Andalucía y España incluyen todos los datos de las capitales, presentan esperanzas de vida menores que los correspondientes a las zonas rurales de esas mismas unidades territoriales. La menor esperanza de vida que muestran los datos de Almería, en relación con Vera, corrobora la sobremortalidad urbana constatada por numerosos estudios (García Gómez, 2016). Esta circunstancia se va reduciendo a nivel nacional, a partir de 1930, especialmente en lo que a salud infantil se refiere (Pérez Moreda et al., 2015: 211-248).

El principal razonamiento para explicar estas diferencias en la mortalidad entre las capitales de provincia y el resto de población ha sido la existencia de penalización urbana o urban penalty. Este término describe la sobremortalidad urbana durante la revolución industrial observada en numerosas ciudades y se ha relacionado con factores como el medio ambiente, el hacinamiento, las migraciones, así como con ciertos estilos de vida que solían darse en las urbes (Szreter y Mooney, 1998; Reher, 2001; Breschi et al., 2011; Escudero y Nicolau, 2014; Escudero et al., 2015). Sin embargo, en los últimos años algunos estudios han señalado que parte de la sobremortalidad urbana puede deberse a imprecisiones en los registros y al efecto composición o selección. Es decir, que podría estar provocado por una mayor presencia en las ciudades de determinados subgrupos como enfermos, presidiarios, huérfanos o personas dependientes con tasas de mortalidad normalmente superiores al resto de población (Pujadas Mora, 2009: 196-198; Revuelta Eugercios y Ramiro-Fariñas, 2016).

Como comentamos en el apartado metodológico, el Índice de Gini no permite responder a las preguntas acerca de las diferencias entre clases sociales. Esto se debe a que mide la mayor o menor dispersión o concentración de los valores. Sin embargo, hemos decido incluirlo puesto que, al computar para su estimación al conjunto de los fallecidos 
(Wagstaff et al., 1991), nos permite observar uno de los rasgos principales de la transición demográfica: la reducción de la mortalidad infantil ${ }^{12}$.

Gráfico 3. Índice de Gini a partir de las edades de fallecimiento

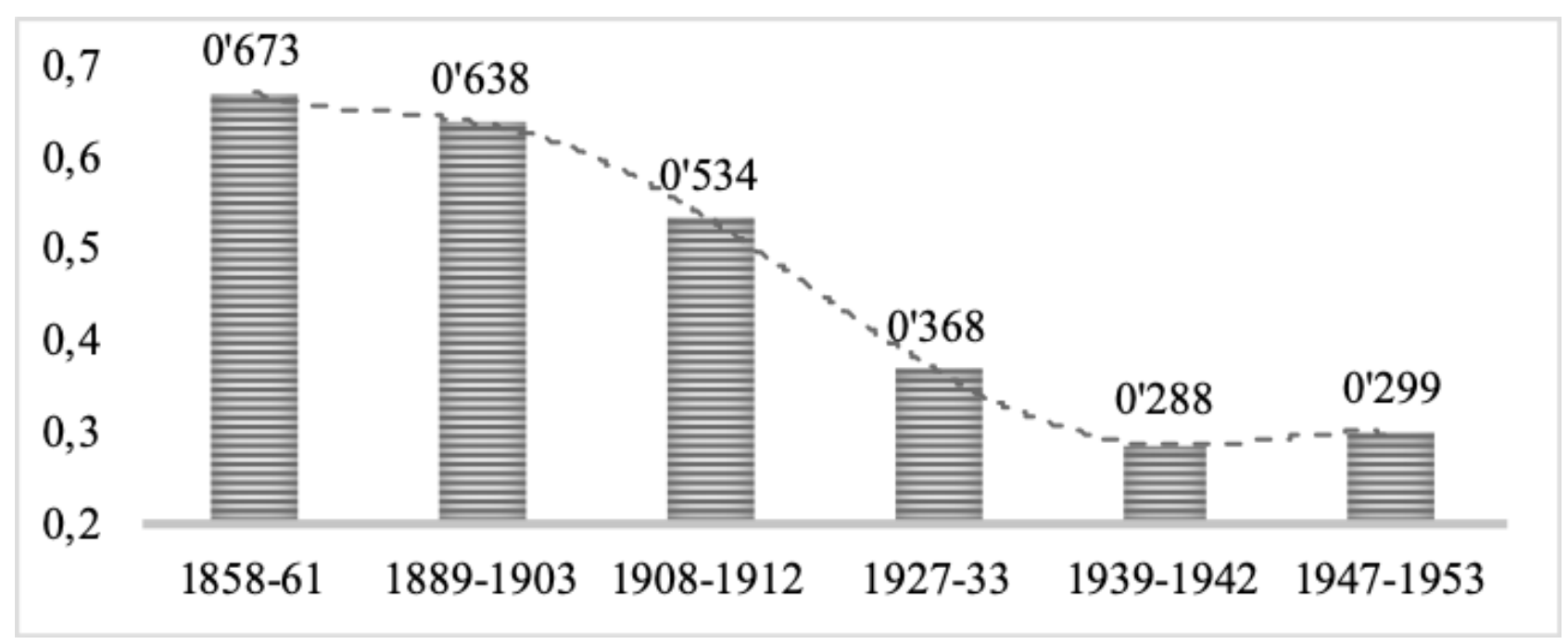

Fuente: Elaboración propia a partir de los libros de defunciones anteriormente referenciados.

Los cálculos muestran una reducción de su valor desde el primer subperiodo (18581861) hasta el penúltimo (1939-1942). Entre este año y el último subperiodo de la serie el valor del indicador aumenta ligeramente. Observamos cómo el mayor ritmo de reducción de la desigualdad se dio entre 1900 y 1940. Esta disminución en los valores del indicador que se produce entre finales del siglo XIX y el primer tercio del siglo XX refleja la notable reducción de la mortalidad infantil y juvenil que se produjo en este periodo y la consecuente concentración de la mortalidad en las edades adultas ${ }^{13}$. El ligero repunte que se produce en la década de los 50 podría ser reflejo del mayor ritmo de mejora en los niveles de supervivencia experimentado por las clases más aventajadas en relación con la clase baja descrito en el siguiente apartado.

\section{LA DESIGUALDAD EN LA MORTALIDAD}

En la tabla 2 mostramos la comparación entre las tasas de mortalidad estandarizadas de la categoría que hemos definido como clase media-alta y las de la clase baja. Igualmente se incluyen las diferencias de mortalidad entre géneros, desagregados por tramos de edad.

12 El Índice de Gini puede tomar valores que van de 0 a 1. Un valor cercano a 1 implicaría grandes diferencias en la edad de fallecimiento entre individuos. Un valor del indicador igual a 0 indicaría que todas las personas fallecen exactamente con la misma edad.

13 Precisamente, durante esta fecha se produjeron los mayores avances en la reducción de la mortalidad infantil pasando la probabilidad de muerte de fallecer durante los primeros 10 años de vida $\left({ }_{10} q_{0}\right)$ de suponer un $477^{\prime} 1 \%$ en 1900 a un $116^{\prime} 7 \%$ en 1951. Nuestros resultados muestran una evolución de la mortalidad infantil en línea con los resultados mostrados por Ramiro-Fariñas y Sanz Gimeno (2000) para varias regiones de España Central durante este mismo periodo. 
Tabla 2. Diferencias de mortalidad entre sexos y categorías socioeconómicas en Vera. Tasas de mortalidad en \%o.

\begin{tabular}{|l|c|c|c|c|c|c|}
\hline & $\begin{array}{c}1858- \\
1861\end{array}$ & $\begin{array}{c}1899- \\
1903\end{array}$ & $\begin{array}{c}1908- \\
1912^{\dagger}\end{array}$ & $\begin{array}{c}1927- \\
1933\end{array}$ & $\begin{array}{c}1939- \\
1942^{\dagger}\end{array}$ & $\begin{array}{c}1947- \\
1953\end{array}$ \\
\hline Mujeres >15* & $21^{\prime} 7$ & $17^{\prime} 5$ & $21^{\prime} 7$ & $11^{\prime} 9$ & $19^{\prime} 3$ & $9^{\prime} 5$ \\
\hline De 15 a 39 años & $11^{\prime} 7$ & $7^{\prime} 5$ & $10^{\prime} 0$ & $4^{\prime} 9$ & $5^{\prime} 3$ & $2^{\prime} 5$ \\
\hline De 40 a 59 años & $19^{\prime} 2$ & $10^{\prime} 5$ & $18^{\prime} 9$ & $8^{\prime} 4$ & $13^{\prime} 4$ & $7^{\prime} 5$ \\
\hline 60 años o más & $65^{\prime} 4$ & $77^{\prime} 8$ & $85^{\prime} 2$ & $63^{\prime} 3$ & $114^{\prime} 6$ & $55^{\prime} 7$ \\
\hline Varones >15* & $30^{\prime} 4$ & $18^{\prime} 4$ & $23^{\prime} 0$ & $16^{\prime} 1$ & $23^{\prime} 2$ & $11^{\prime} 2$ \\
\hline De 15 a 39 años & $15^{\prime} 2$ & $7^{\prime} 3$ & $7^{\prime} 2$ & $6^{\prime} 3$ & $3^{\prime} 1$ & $2^{\prime} 6$ \\
\hline De 40 a 59 años & $24^{\prime} 5$ & $17^{\prime} 2$ & $20^{\prime} 1$ & $13^{\prime} 7$ & $20^{\prime} 0$ & $7^{\prime} 7$ \\
\hline 60 años o más & $97^{\prime} 0$ & $76^{\prime} 0$ & $91^{\prime} 9$ & $76^{\prime} 9$ & $141^{\prime} 2$ & $65^{\prime} 8$ \\
\hline Clase Media Alta (HISCLASS 1-7) & $15^{\prime} 6$ & $12^{\prime} 9$ & $18^{\prime} 3$ & $16^{\prime} 1$ & $17^{\prime} 3$ & $7^{\prime} 2$ \\
\hline Clase Baja (HISCLASS 8-12) & $29^{\prime} 4$ & $22^{\prime} 6$ & $27^{\prime} 3$ & $16^{\prime} 6$ & $30^{\prime} 1$ & $16^{\prime} 7$ \\
\hline Ratio Baja / Media Alta (\%) & $188^{\prime} 0$ & $175^{\prime} 5$ & $149^{\prime} 8$ & $103^{\prime} 5$ & $173^{\prime} 9$ & $231^{\prime} 3$ \\
\hline $\begin{array}{l}\text { Varones sin ocupación en libros de } \\
\text { defunciones }\end{array}$ & $\begin{array}{c}16^{\prime} 6 \\
\%\end{array}$ & $22^{\prime} 9$ & $1^{\prime} 2 \%$ & $2^{\prime} 6 \%$ & $1^{\prime} 2 \%$ & $4^{\prime} 9 \%$ \\
\hline
\end{tabular}

Fuente: Elaboración propia a partir de los datos de los censos y los libros de defunciones parroquiales. Los periodos señalados con una "†" incluyen años de crisis de mortalidad: fiebre tifoidea en el 1908-1912 y la posguerra en el periodo 1939-1943. El símbolo "*” significa que la tasa está estandarizada a partir de la distribución de la población española en 1900.

Los datos de mortalidad estandarizados de los hombres mayores de 15 años son superiores a los de las mujeres en todos los periodos, observándose unas tendencias de ambos géneros bastante similares. Sin embargo, esta sobremortalidad masculina se debe principalmente a las mayores tasas experimentadas a partir de la edad de 40 años. Como diversos estudios han acreditado, la ventaja femenina en relación con la mortalidad desaparece en las edades fértiles, encontrándonos en ese tramo incluso con tasas superiores en las mujeres, provocadas por la mortalidad asociada al parto (Loudon, 1992; Livi Bacci, 1993: 148). Este diferencial de sobremortalidad femenina en edades fértiles se mantiene durante el primer tercio del siglo XX hasta 1930 cuando, al igual que sucede para el ámbito nacional, las tasas de mortalidad masculinas superan a las femeninas (Pérez Moreda et al., 2015: 120-126). Sin embargo, esta tendencia se ve temporalmente truncada con el repunte de la mortalidad en el periodo de posguerra. No será hasta la década de 1950 cuando las tasas masculinas vuelvan a superar a las femeninas en este tramo de edad. Este hecho podría reflejar un retraso en las mejoras sanitarias relacionadas con la maternidad en la ciudad de Vera respecto a la media nacional (González Canalejo, 2005: 
151-174). Aunque también podría venir provocado por un posible subregistro de defunciones masculinas consecuencia del desplazamiento de los varones al frente y del fallecimiento de un porcentaje de ellos fuera de la ciudad.

En ambos sexos, el valor máximo en las tasas se produce en el periodo 18581861. Sin embargo, el segundo valor más alto difiere según el género. En los varones se encuentra en los años de la posguerra (1939-1942) mientras que, para las mujeres, ocurre durante el episodio de fiebre tifoidea que experimentó la ciudad de Vera durante el año 1911. El comportamiento diferencial es coherente con las conclusiones de Goldin y Lleras-Muney (2018), en las que se sostiene que las enfermedades infecciosas tuvieron una mayor incidencia en la mortalidad femenina. Ambos sexos experimentan las menores tasas durante el último subperiodo estudiado 1947-1953. Este menor nivel de mortalidad es reflejo de la caída tendencial propia de la transición demográfica en un momento en el que España está cerca de completar su paso a un régimen demográfico moderno (Pérez Moreda et al., 2015).

La tendencia en la evolución de las tasas de mortalidad del conjunto de categorías ocupacionales refleja lógicamente la reducción que, a nivel agregado, hemos observamos en el gráfico 1. Sin embargo, en la tabla 2 advertimos que la caída se produce a un ritmo diferente entre las distintas categorías socioeconómicas. Al analizar dicha tabla es importante tener presente que, en los subperiodos 1908-12 y 1939-42, el repunte en los valores de mortalidad viene explicado fundamentalmente por la epidemia de fiebre tifoidea de 1911, en el primer caso y por la sobremortalidad asociada a las condiciones de posguerra, en el segundo.

Gráfico 4. Mortalidad de la categoría socioeconómica baja en relación con la media-alta

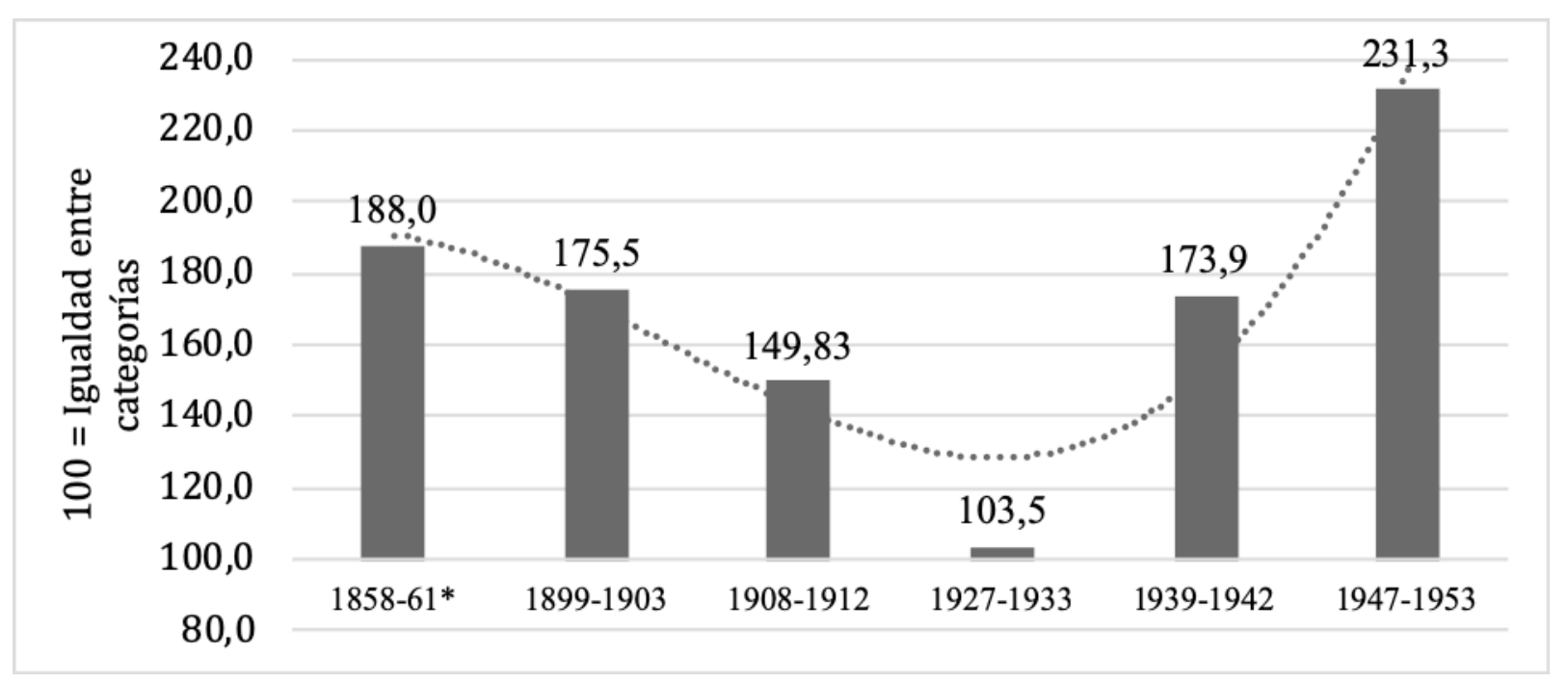

Fuente: Elaboración propia a partir de los distintos censos y libros de defunciones parroquiales anteriormente referenciados.

En cuanto a los resultados comparados de mortalidad entre clases sociales, se observa, que los niveles más álgidos de desigualdad en el fallecimiento entre clases se encuentran a principio y al final del periodo estudiado, específicamente en los subperiodos 1858-1861 y 1947-1953. Así, aunque el periodo estudiado comienza con una elevada desigualdad social en la mortalidad, este indicador se reduce notablemente a partir de la primera década del siglo XX, alcanzando en 1930 los niveles de desigualdad más bajos de toda la serie. Esta 
convergencia se ve truncada con la Guerra Civil y el periodo de posguerra. En el último subperiodo estudiado (1947-1953) la desigualdad es mayor que en el anterior (1939-1942) como consecuencia del mayor ritmo de reducción de la mortalidad experimentado por las clases más pudientes en contraposición a la menor reducción de la tasa de mortalidad de las categorías de menor estatus socioeconómico. Es posible que los déficits nutricionales del periodo de la Guerra Civil y la posguerra y la mayor prevalencia de enfermedades infecciosas durante estos años hayan influido, en mayor medida, en aquellos individuos clasificados en el estatus socioeconómico bajo. Esto ha podido provocar, como distintos estudios han señalado (Bengtsson y Lindström, 2000; Floud, Fogel, Harris y Hong, 2011: 105), unos niveles de mortalidad superiores en las décadas subsiguientes.

Los valores en el subperiodo 1927-1933 muestran una práctica ausencia de desigualdad. Resultados similares han sido mostrados en distintos estudios internacionales y, en la mayoría de los casos, se han justificado aludiendo a factores de comportamiento (hábitos poco saludables) como elemento compensador de la teórica ventaja en el nivel de vida (Edvinsson y Broström, 2017). En nuestro caso es probable que tanto las mejoras nutricionales como los avances higiénicos y en la salud pública coadyuvaran a la reducción de la desigualdad en las primeras décadas del siglo XX.

Con la Ley de Saneamiento y Reforma Interior de las Poblaciones de 1895 y, especialmente con la aprobación del Estatuto Municipal de 1924, se impulsó en Vera "la realización de obras de reforma para adaptar la ciudad y conformarla con arreglo a los nuevos planteamientos higiénico-sanitarios" (Cano, 2015: 133-137). Así mismo, la caída poblacional entre 1910 y 1930 (de 8.651 habitantes a 5.336) pudo facilitar el acceso a la vivienda para las clases populares, reduciendo el hacinamiento. Durante este periodo se experimentaron, en la provincia de Almería y especialmente en la ciudad de Vera, elevadas tasas migratorias, tanto temporales como definitivas. Éstas reflejaron los problemas sufridos por los sectores exportadores y el efecto llamada que provocó el proceso de industrialización y urbanización en otras regiones españolas y las migraciones exteriores hacia Argelia y Sudamérica (Sánchez Picón, 2005). Por desgracia, las condiciones de posguerra revirtieron el efecto de las mejoras sanitarias e higiénicas provocando la reaparición de enfermedades infecciosas, cuya frecuencia se había visto reducida considerablemente en los años anteriores.

El elevado nivel de mortalidad mostrado por los individuos de menor estatus socioeconómico durante el subperiodo 1858-1861 es coherente con la caída de la talla de los mozos nacidos entre la segunda mitad del siglo y la década de 1870 mostrada por Martínez-Carrión $(2005,2006)$ para esta misma ciudad. La evolución del diferencial de mortalidad entre clases durante las primeras décadas del siglo XX parece coincidir también con la "modesta mejora en la nutrición [...] y el impulso de una serie de mejoras sociales y de la creciente preocupación por la salud" que, a nivel nacional, describen Pérez Moreda et al. (2015: 133-137). Del mismo modo, el incremento en la desigualdad se correspondería con la nula mejora nutricional para la mayoría de la población entre 1936 y los primeros años de la década de 1950, que también se vio reflejada en un aumento de las diferencias de estatura entre los trabajadores no cualificados y el resto de los quintos de esas generaciones (Martínez-Carrión, 2005, 2006). Debemos tener en cuenta que la transición nutricional no fue un proceso homogéneo entre categorías socioeconómicas. Estudios como el de Medina-Albaladejo y Calatayud (2019) muestran que la evolución de las dietas típicas de los distintos grupos sociales fue muy diferente. Mientras que las de las clases altas ya incluían a mediados del siglo XIX los alimentos que caracterizaron la transición nutricional, la de las clases populares no permitía cubrir de forma adecuada sus necesidades básicas hasta la década de 1920. Situación que volvió a recrudecerse, especialmente para estas últimas, en los años de la posguerra y autarquía (Ibáñez Domingo, 2014). 
En la tabla 2 hemos representado con cursiva aquellos valores desagregados por clases sociales para los que, debido a la mayor prevalencia de subregistro de las ocupaciones en los libros de defunciones, debemos ser especialmente cautelosos. El subregistro en los registros entre 1898-1902 (23'9 \%) es significativamente superior al promedio de los cuatro subperiodos siguientes (3'9 \%). Además, la subinscripción de oficios en ese censo es la menor de todas. La coincidencia de ambas circunstancias es la principal explicación a los llamativamente reducidos niveles de mortalidad de ambas categorías socioeconómicas. Si tomamos como hipótesis que el subregistro tiene la misma intensidad en términos relativos en una clase social que en otra, los resultados de la ratio de desigualdad serían válidos. Sin embargo, se podría justificar que el subregistro pudiese estar sesgado hacia una clase social o hacia la otra, razón por la cual advertimos de la cautela con la que se deben interpretar dichos resultados.

Otros factores que pueden haber influido en los niveles de desigualdad social podrían estar relacionados con la forma en que hemos tratado los datos, puesto que no hemos considerado categoría de análisis la clase social de la familia sino la ocupación de la persona. En este sentido, la probable existencia de mayores niveles de mortalidad infantil entre las clases bajas podría provocar una reducción de los niveles de desigualdad en la mortalidad adulta derivadas de un efecto selección durante las edades pre-adultas. Tampoco puede obviarse el efecto selectivo que podría haberse producido en aquellas ocupaciones que requieren de mayor esfuerzo físico como los jornaleros. En este caso, la selección vendría explicada porque las probabilidades de desempeñar un trabajo con los requerimientos físicos elevados serían mayores para personas que tuvieran una buena condición física. Así, es probable que no se hayan tenido en cuenta algunos de los individuos de las clases bajas que estuvieran enfermos o en peores condiciones físicas, al no figurar como jornaleros ni en los libros censales ni en los parroquiales. Por el contrario, en el otro grupo de la población, el hecho de no tener unas condiciones de salud adecuadas no impediría que la persona figurase como propietario o estudiante (Jaadla, Puur y Rahu, 2017: 18; Livi Bacci, 1993: 184-191).

Otro factor que podría introducir un sesgo serían las elevadas tasas de emigración temporal existentes en el municipio de Vera, según se desprende de los registros de pasaportes del XIX. En los estudios realizados, se registran en los años 1849-1854 promedios anuales de expedición de entre 700 y 1.000 pasaportes, en su mayor parte gratuitos, sugiriendo el bajo nivel de renta de los solicitantes. Estas cifras suponen un elevado porcentaje (superior al $15 \%$ ) de la población censada y equivalente a más de una tercera parte de la población jornalera (Sánchez Picón, 1988). Ello significa que la mortalidad producida durante el periodo de emigración en dicha franja de edad no constaría en los libros de defunciones parroquiales de Vera, al haber fallecido en otros lugares, a pesar de estar incluidos en el censo (Revuelta Eugercios y Ramiro-Fariñas, 2016). En todo caso, esta hipótesis exige estudiar la distribución en edad y profesión de los emigrantes temporales.

\section{CONCLUSIONES}

Nuestros resultados muestran una clara asociación, en el territorio estudiado y durante la mayoría de los subperiodos analizados, entre la probabilidad de muerte y el contexto sociolaboral. En relación con la evolución de las diferencias de mortalidad por categorías socioeconómicas, se ha observado una progresiva reducción de la desigualdad de fallecimiento desde el año 1858 hasta los años 30 del siglo XX, progresión que se trunca dramáticamente en los años de la posguerra. El comportamiento de la mortalidad 
desagregado por categorías socioeconómicas parece estar en línea con la evolución de los factores nutricionales y los relacionados con los avances higiénicos y en la salud pública.

Realizando una lectura de nuestros resultados a partir de las principales visiones sobre la evolución de la desigualdad social en la mortalidad, podemos concluir que no coinciden con la hipótesis de la constancia puesto que no en todos los subperiodos hemos constatado niveles de mortalidad significativamente superiores de la clase baja. Sin embargo, tampoco encajan exactamente con el modelo propuesto por Antonovsky (1967) debido a las notables diferencias observadas en las primeras fases de la transición demográfica.

La comparación de nuestros resultados con los obtenidos por otros estudios invita a profundizar en el análisis de las posibles causas que han provocado datos tan dispares en los distintos países y modelos de desarrollo. Nuestros resultados parecen estar más en la línea de los mostrados en estudios sobre economías mediterráneas. Este hecho podría estar relacionado, en parte, con las diferencias salariales que muestra Allen (2001) en relación con el mínimo de subsistencia en la primera fase de la transición demográfica.

Sinembargo, la metodología utilizada no nos ha permitido aislar el impacto de los distintos determinantes de la mortalidad en cada momento, como los asociados a las diferencias de comportamiento, a la distribución geográfica de los domicilios y sus características, a las dietas o a los salarios. Por otro lado, al tratarse de un estudio transversal, las diferencias en las tasas de mortalidad por tramos de edad pueden estar asociadas tanto a un efecto edad o como a un efecto generación, siendo imposible precisar con exactitud la importancia de cada uno.

Por último, advertimos que los resultados presentados deben acogerse con precaución, al estar circunscritos a una ciudad y unas circunstancias particulares. En este sentido, sería interesante ampliar el número de ciudades objeto de estudio para constatar, entre otras cosas, las diferencias en la mortalidad que han surgido durante el desarrollo de la transición demográfica en distintas regiones y modelos productivos. No obstante, creemos que el análisis que se hace a partir del tratamiento sistemático de los fallecidos a lo largo de un siglo aporta nuevas evidencias para el caso español que se sitúan en la línea de algunos de los recientes debates que, desde diferentes perspectivas, se han planteado sobre el estudio histórico de la desigualdad.

\section{BIBLIOGRAFÍA}

Allen, R. (2001), "The great divergence in European wages and prices from the middle ages to the First World War", Explorations in Economic History, 38(4), pp. 411-447.

Antonovsky, A. (1967), "Social Class, Life Expectancy and Overall Mortality Reviewed work", The Milbank Memorial Fund Quarterly, 45(2), pp. 31-73.

Artola, M., Bernal, A. y Contreras, J. (1978), El latifundio: Propiedad y explotación, ss. XVIII$X X$, Madrid, Servicio de Publicaciones Agrarias.

Banco de España (1900), Memoria sobre la riqueza y posible desenvolvimiento mercantil de la región Norte de la provincia de Almería y demostración de la utilidad que podría reportar el establecimiento de una sucursal del Banco de España en Cuevas, Almería.

Bengtsson, T. (2004), "Living Standards and Economic Stress", en T. Bengtsson, C. Campbell, C. y J. Z. Lee, Life under Pressure: Mortality and Living Standards in Europe and Asia, 1700-1900, Cambridge, MA, MIT Press, pp. 27-59.

Bengtsson, T., Campbell, C. y Lee, J. Z. (2004), Life under pressure: mortality and living standards in Europe and Asia, 1700-1900, Cambridge, MA, MIT Press.

Bengtsson, T. y Lindström, M. (2000), "Childhood misery and disease in later life: The effects on mortality in old age of hazards experienced in early life, southern Sweden, 17601894", Population Studies, 54(3), pp. 263-277. 
Bengtsson, T. y Van Poppel, F. (2011), "Socioeconomic inequalities in death from past to present: An introduction", Explorations in Economic History, 48(3), pp. 343-356.

Breschi, M., Fornasin, A., Manfredini, M., Mazzoni, S. y Pozzi, L. (2011), "Socioeconomic conditions, health and mortality from birth to adulthood, Alghero 1866-1925", Explorations in Economic History, 48(3), pp. 366-375.

Calot, G. (1999), Un siglo de demografía en Andalucía: La población desde 1900, Sevilla, Instituto de Estadística y Cartografía de Andalucía.

Cámara Hueso, A. D. (2007), Niveles de vida en el medio rural de Andalucía Oriental (1750-1950), Granada, Universidad de Granada.

Cano, L. (2015), Historia Urbana de Vera. Castillo en la traza, Granada, Universidad de Granada.

Clark, G. (2008), A Farewell to Alms: a brief economic history of the world, Princeton, Princeton University Press.

Clouston, S. A. P., Rubin, M. S., Phelan, J. C. y Link, B. G. (2016), "A Social History of Disease: Contextualizing the Rise and Fall of Social Inequalities in Cause-Specific Mortality", Demography, 53(5), pp. 1631-1656.

Domínguez Martín, R. y Guijarro Garvi, M. (2000), "Evolución de las disparidades espaciales del bienestar en España, 1860-1930. El Índice Físico de Calidad de Vida", Revista de Historia Económica / Journal of Iberian and Latin American Economic History, 18(01), pp. 109-137.

Dopico, F. y Reher, D. S. (1998), El declive de la mortalidad en España, 1860-1930, Asociación de Demografía Histórica.

Edvinsson, S. y Broström, G. (2017), "Life course and long-term perspectives of social inequality in mortality among elderly and adults in Northern Sweden 1801-2013", divaportal.org.

Elder, G. y Pellerin, L. (1998), "Linking history and human lives", Methods of life course research: Qualitative and quantitative approaches, pp. 264-294.

Escudero, A. y Nicolau, R. (2014), "Urban penalty: nuevas hipótesis y caso español (18601920)", Historia Social, (80), pp. 9-33.

Escudero, A., Pérez de Perceval, M. Á. y Sánchez Picón, A. (2015), "Urban environmental degradation and the standard of living: the case of the Spanish mining industry (18701930)", Continuity and Change, 30(3), pp. 395-421.

Floud, R., Fogel, R., Harris, B. y Hong, S. (2011), The changing body: Health, nutrition, and human development in the western world since 1700, Cambridge, Cambridge University Press.

Fox, A. J, Goldblatt, P. O. y Jones, D. R. (1985), "Social class mortality differentials: artefact, selection or life circumstances?", Journal of Epidemiology and Community Health, (39), pp. 1-8.

García Gómez, J. J. (2016), "'Urban penalty' en España: el caso de Alcoy (1857-1930)", Historia Industrial, 25(63), pp. 49-78.

Goerlich Gisbert, F. J. y Pinilla Pallejà, R. (2006), Esperanza de vida en España a lo largo del siglo XX Las tablas de mortalidad del Instituto Nacional de Estadística, Bilbao, Fundación BBVA.

Goldin, C. y Lleras-Muney, A. (2018), “XX>XY?: The Changing female advantage in life expectancy", NBER Working Paper Series, 24716, pp. 1-49

González Canalejo, C. (2005), Asistencia sanitaria, género y cuestión social en Almería (1857-1930), Almería, Universidad de Almería.

González García, A. (2013), "Avances y tendencias actuales en el estudio de la pandemia de gripe de 1918-1919", Vínculos de Historia, 2, pp. 309-330.

Haines, M. R. (2001), "The Urban Mortality Transition in the United States, 1800-1940", 
NBER Working Paper Series, 3, pp. 1-39.

Harris, B. (2008), "Gender, health, and welfare in England and Wales since industrialization", Research in Economic History, 26, pp. 157-204.

Ibáñez Domingo, M. (2014), "Estómagos vacíos. La miseria de las mujeres vencidas en la inmediata posguerra", Vínculos de Historia, 3, pp. 302-32

Instituto de Estadística y Cartografía de Andalucía (2004), Evolución histórica de la población de Andalucía, Sevilla, Instituto de Estadística y Demografía.

Jaadla, H., Puur, A. y Rahu, K. (2017), "Socioeconomic and cultural differentials in mortality in a late 19th century urban setting: A linked records study from Tartu, Estonia, 18971900", Demographic Research, 36(1), pp. 1-40.

Kesztenbaum, L. y Rosenthal, J.-L. (2016), "The Democratization of Longevity: How the Poor Became Old in Paris, 1880-1913", en D. Ramiro Fariñas y M. Oris (eds.), New Approaches to Death in Cities during the Health Transition, New York, Springer International Publishing, pp. 137-154.

Van Leeuwen, M. y Maas, I. (2011), Hisclass: A Historical International Social Class Scheme, Leuven, Universitaire Pers Leuven.

Van Leeuwen, M., Maas, I. y Miles, A. (2002), HISCO: Historical international standard classification of occupations, Leuven, Universitaire Pers Leuven.

Link, B. G. y Phelan, J. C. (1995), "Social Conditions as Fundamental Causes of Disease", Journal of Health and Social Behavior, 35, pp. 80-94.

- (1996), "Understanding sociodemographic differences in health--the role of fundamental social causes", American Journal of Public Health, 86(4), pp. 471-473.

- (2002), "McKeown and the Idea That Social Conditions Are Fundamental Causes of Disease", American Journal of Public Health, 92(5), pp. 730-732.

Livi Bacci, M. (1990), Historia mínima de la población mundial, Barcelona, Ariel.

- (1993), Introducción a la demografía, Barcelona, Ariel.

Llorca, J., Prieto Salceda, D. y Delgado-Rodríguez, M. (2000), "Medición de las desigualdades en la edad de muerte: cálculo del índice de gini a partir de las tablas de mortalidad", Revista Española de Salud Pública, 74(1), pp. 5-12.

Loudon, I. (1992), Death in childbirth: an international study of maternal care and maternal mortality, 1800-1950, Oxford, Clarendon Press.

Marmot, M. (2004), "Status síndrome", Significance, 1(4), pp. 150-154.

Martínez-Carrión, J. M. (2005), "Estatura, salud y nivel de vida en la minería del sureste español, 1830-1936", Revista de Demografía Histórica, 23(1), pp. 1-38.

Martínez Carrión, J. M. (2006), "Niveles de Vida en la Minería Española. 1840-1936", en M. A Pérez De Perceval Verde, M. A. López-Morell, y A. Sánchez Rodríguez (eds.), Minería y desarrollo económico en España, Madrid, Editorial Síntesis, pp. 237-256.

Martínez-Carrión, J. M. y Pérez Castejón, J. J. (2002), "Growing with inequality — Creciendo con desigualdad. Niveles de vida biológicos en la España rural mediterránea desde 1840", en J. M. Martínez-Carrión (ed.), El Nivel de Vida en la España Rural, Siglos XVIII-XX, Alicante, Universidad de Alicante, pp. 405-460.

Medina-Albaladejo, F. y Calatayud, S. (2019), "Inequality during the nutritional transition: Hospital diets in Mediterranean Spain (Valencia, 1853-1923)", DT-AEHE, 1909, pp. 1-26.

Mignon, C. (1982), Campos y campesinos de la Andalucía mediterránea, Madrid, Ministerio de Agricultura, Servicio de Publicaciones Agrarias.

Nadal, J. (1984), La población española: siglos XVI a XX, Barcelona, Ariel.

Nicolau, R. (2005), "Población, salud y actividad", en A. Carreras, y X. Tafunell (eds.), Estadísticas históricas de España siglos xix-xx, Bilbao, Fundación BBVA, pp. 77-154. 
Pamuk, E. R. (1985), "Social Class Inequality in Mortality From 1921 to 1972 in England and Wales", Population Studies, 39(1), pp. 17-31.

Pareja Alonso, A. (2006), "La actividad laboral y productiva de las mujeres bilbaínas en 1900. Una propuesta metodológica para su recuperación", Vasconia, 35, pp. 201-219.

Parejo Barranco, J. A. (2006), "De la región a la ciudad. Un nuevo enfoque de la historia industrial española contemporánea", Revista de historia industrial, (30), pp. 53-102.

Pérez De Perceval Verde, M. Á., Martínez-Carrión, J. M. y Martínez-Soto, Á. P. (2016), "Biological welfare and inequality during the mining boom: Rio Tinto, 1832-1935", Revista de Historia Industrial, 25(64), pp. 149-181.

Pérez Moreda, V., Reher, D. S. y Sanz Gimeno, A. (2015), La conquista de la salud: mortalidad y modernización en la España contemporánea, Madrid, Marcial Pons Historia.

Pressat, R. (1993), El análisis demográfico: conceptos, métodos, resultados, Madrid, Fondo de Cultura Económica.

Pujadas Mora, J. M. (2009), L'evolució de la mortalitat infantil i juvenil a la ciutat de Palma (Mallorca, 1838-1960), Universitat de les Illes Balears.

Pujadas Mora, J. M., Romero Marín, J. y Villar Garruta, C. (2014), "Propuestas metodológicas para la aplicación de HISCO en el caso de Cataluña, siglos XV-XX", Revista de Demografía Histórica, 32(1), pp. 181-219.

Ramiro-Fariñas, D. y Sanz Gimeno, A. (2000), "Childhood mortality in Central Spain, 17901960: Changes in the course of demographic modernization", Continuity and Change, 15(2), pp. 235-267.

Recaño, J. y Esteve, A. (2006), "Revisitando Garcia Faria: Un estudio de los factores espaciales y medioambientales de la mortalidad en la Barcelona de finales del siglo XIX", Revista de Demografía Histórica, XXIV (1), pp. 121-180.

Reher, D. S. (2000), "La investigación en demografía histórica: pasado, presente y futuro", Revista de demografía histórica, 18(2), pp. 15-78.

Reher, D. S. (2001), "In search of the 'urban penalty': exploring urban and rural mortality patterns in Spain during the demographic transition", International Journal of Population Geography, 7(2), pp. 105-127.

Reques, L., Miqueleiz, E., Giráldez-García, C., Santos, J. M., Martínez, D. y Regidor, E. (2015), "Patrones geográficos de la mortalidad y de las desigualdades socioeconómicas en mortalidad en España", Revista Española de Salud Pública, 89, pp. 137-147.

Revuelta Eugercios, B. y Ramiro-Fariñas, D. (2016), "Understanding Infant Mortality in the City: Exploring Registration and Compositional Effects. Madrid, 1905-1906", en D. Ramiro Fariñas y M. Oris (eds.), New Approaches to Death in Cities during the Health Transition, New York, Springer, pp. 19-42.

Robles González, E., García Benavides, F. y Bernabeu-Mestre, J. (1996), "La transición sanitaria en España desde 1900 a 1990", Revista Española de Salud Pública, 70(2), pp. 221-233.

Sánchez Aguilera, D. (1996), "Las diferencias territoriales de la mortalidad en Andalucía a fines del siglo XIX", Revista de Demografía Histórica, 14(2), pp. 151-172.

Sánchez Picón, A. (1988), "Marchar a las Andalucías. Un episodio migratorio en la Almería del siglo XIX", en Homenaje al Padre Tapia, Almería, Unicaja.

Sánchez Picón, A. (1992), La integración de la economía almeriense en el mercado mundial (1776-1936), Almería, Instituto de Estudios Almerienses de la Diputación Provincial de Almería.

- (2005), "De frontera a milagro. La conformación histórica de la economía almeriense", en J. Molina Herrera (ed.), La economía de la provincia de Almería, El Ejido, Cajamar, pp. 43-86. 
- (2011), "Ejes de desarrollo en el Sureste y la Andalucía mediterránea (1800-2000)", Historia Contemporánea, 42, pp. 261-302.

Sarasua, C. (2019), "Women's work and structural change: occupational structure in eighteenth-century Spain", The Economic History Review, 72(2), pp. 481-509.

Schenk, N. y Van Poppel, F. (2011), "Social class, social mobility and mortality in the Netherlands, 1850-2004", Explorations in Economic History, 48(3), pp. 401-417.

Schumacher, R. y Oris, M. (2011), "Long-term changes in social mortality differentials, Geneva, 1625-2004", Explorations in Economic History, 48(3), pp. 357-365.

Szreter, S. y Mooney, G. (1998), "Urbanization, Mortality, and the Standard of Living Debate: New Estimates of the Expectation of Life at Birth in Nineteenth-century British Cities", The Economic History Review, 51(1), pp. 84-112.

Vázquez, E., Camaño, F., Silvi, J. y Roca, A. (2003), "La tabla de vida: una técnica para resumir la mortalidad y la sobrevivencia", Boletín Epidemiológico, 24(4), pp. 6-10.

Wagstaff, A., Paci, P. y Van Doorslaer, E. (1991), "On the measurement of inequalities in health", Social Science and Medicine, 33(5), pp. 545-557.

Williamson, J. G. (1990), Coping with City Growth during the British Industrial Revolution, Cambridge, Cambridge University Press.

Winkler, W. E. (1999), "The State of Record Linkage and Current Research Problems", Statistical Research Division U.S. Census Bureau.

Zoido, F. y Arroyo, A. (2003), "La población de España", en A. Arrollo (coord.), Tendencias demográficas durante el siglo XX en España, Madrid, Instituto Nacional de Estadística, pp. 17-75. 